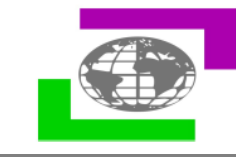

\title{
POLYMORPHISM AND POLYMORPH CHARACTERISATION IN PHARMACEUTICALS
}

Dr Amit Gosar ${ }^{1}$, Dr Sayyed Hussain ${ }^{2}$ and Dr Tabrez Shaikh ${ }^{1}$,

${ }^{1}$ Indoco Remedies Limited, Analytical Research \& Development Department, Navimumbai, 400701, Maharashtra, India.

${ }^{2}$ Sir Sayyed College of Arts, Commerce and Science, P.G. Department of Chemistry, Roshangate, Aurangabad, 431001 Maharashtra, India.

Article Info: Received 03 September 2019; Accepted 30 September. 2019

DOI: https://doi.org/10.32553/jbpr.v8i5.652

Corresponding author: Dr. Tabrez Shaikh

Conflict of interest statement: No conflict of interest

\section{ABSTRACT:}

Polymorphism is the ability of a specific chemical compound to crystallize in more than one crystalline form. Polymorphs had a different arrangement of the molecule in the given crystal lattice and may properties such as packing properties, thermodynamic properties, spectroscopic properties, kinetic properties, surface properties and mechanical properties. Polymorph can be classified in various class such as crystalline, amorphous, hydrate and solvate. Polymorphs are categorized into two types, enantiotropes and monotropes, depending upon their stability with respect to the range of temperatures and pressures. There are various guidelines for the control of polymorphs in drug substances and drug product. Different analytical techniques are used for the detection, quantification and characterisation of the polymorphs in the drug substances and drug products. Control of polymorph in a drug substance and drug product is important for the safety and efficacy of the drug.

Keywords: Polymorphism, classification, guidelines, characterisation, drug substances.

\section{INTRODUCTION}

Polymorphism was first discovered in 1821 by the German chemist Eilhard Mitscherlich and it is a very commonly known phenomenon in the pharmaceutical world which exhibits in crystalline materials. Ostwarld in 1899 stated, "Almost every substance can exist in two or more solid phases provided the experimental conditions are suitable. "Roland Boese, of the University of Duisberg-Essen, Germany, states that 30 to 50 per cent of pharmaceutical compounds exhibit polymorphism. Jean-Paul Garnier, CEO of GlaxoSmithKline said that "About $50 \%$ of drug candidates that enter clinical trials fail due to efficacy and safety concerns, and the remaining $40 \%$ fizzle due to patent concerns and issues like solubility and drug interaction." This shows the importance of polymorphism in Pharmaceutical industries.[1-3].

A crystalline structure consists of a unit cell which is the smallest unit of a crystal which, if repeated, could generate the whole crystal (Figure 1). If the crystal structure of the same chemical compound has two or more arrangements of repeated unit cells, the crystalline structures are called polymorph and the phenomenon is called polymorphism, thus the polymorphism is the ability of a chemical substance to exist in more than one crystalline form which differs in arrangements of the molecules within the crystal lattice.

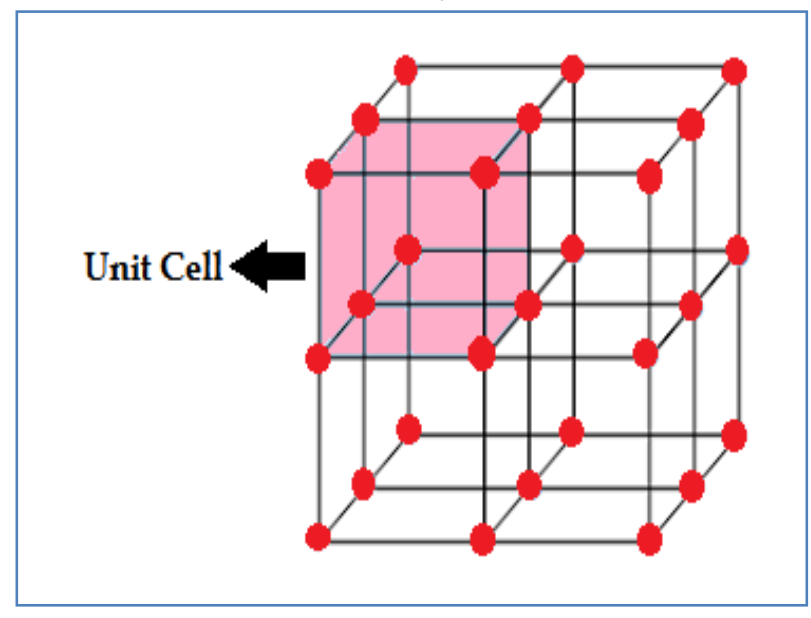

Figure 1: Crystal structure with unit cell 


\section{Type of polymorph}

Based on the pattern of arrangement or associated solvents, polymorph is classified into following different forms [4].

\section{Crystalline form}

These forms are formed when molecules of the drug substance are arranged in repeating order in the given three-dimensional space (Figure 2). Two crystalline forms have a different arrangement of the molecule in the crystal lattice. Crystalline solids are usually highly stable and have well- established properties. Most of the drugs used are having a crystalline form.

\section{Amorphous form}

This type of form consists of randomly arrangements of molecules with a high degree of disorder in the given space and it does not possess a distinguishable crystal lattice (Figure 2). It is also called a non-crystalline solid form. The amorphous state is usually much faster dissolving than the crystalline form and has a variable solubility that is usually higher than the crystalline form. The amorphous form is often less stable than the crystalline form and is usually hygroscopic in nature.

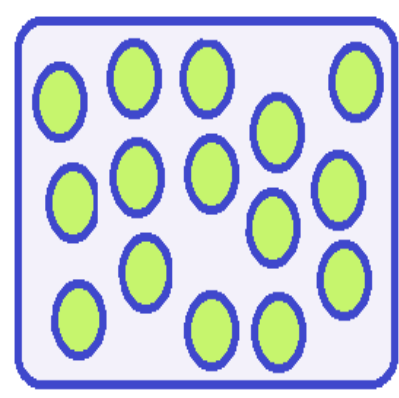

(a)

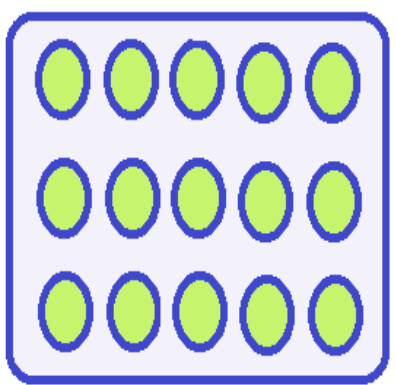

(b)

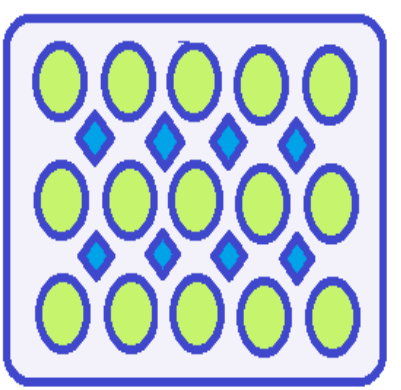

(c)

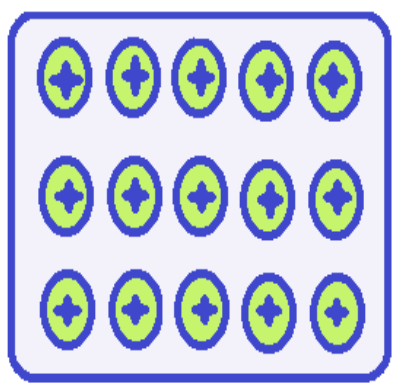

(d)

Figure 2: Type of polymorphic form a) Amorphous, b) crystalline, c) Solvates and d) Co-crystal

\section{Types of Polymorphism}

Polymorphs are categorized into two types, enantiotropes and monotropes, depending upon their stability with respect to the range of temperatures and pressures (Figure 3) [5].

\section{Enantiotropes}

When one polymorphic form changes to another at a fixed temperature (transition temperature) reversibly the phenomenon is called as enantiotropy and the polymorphic forms are said

\section{Solvate form}

When crystal forms containing either stoichiometric or non-stoichiometric amounts of a solvent within the crystal lattice, it is called as solvates (Figure 2). If the incorporated solvent is water, the solvate is called a hydrate. Depending on the number of molecules of water incorporated, this crystal is called as the monohydrate, dihydrate, trihydrate, etc. Hydrates are the most commonly observed class of solvates. Besides water, residual solvents used in the process can get incorporated and forms the solvates.

\section{Co-crystal}

These are formed from crystalline materials which composed of two or more different molecules, typically an active pharmaceutical ingredient and co-crystal (conformers), in the same crystal lattice (Figure 2). These are bonded with each other through non-covalent bonding such as hydrogen bonding, van-der-Waals interactions or $\Pi$ interactions. Co-crystals have defined stoichiometry. These materials are designed through crystal engineering for better solubility and stability of the drug. 
monotropy and the two polymorphs are said to be monotropes of each other. The metastable form changes to the stable form at all temperature and the change is not reversible. As a result, all other metastable polymorphs have no region of stability anywhere on a pressure-temperature diagram.

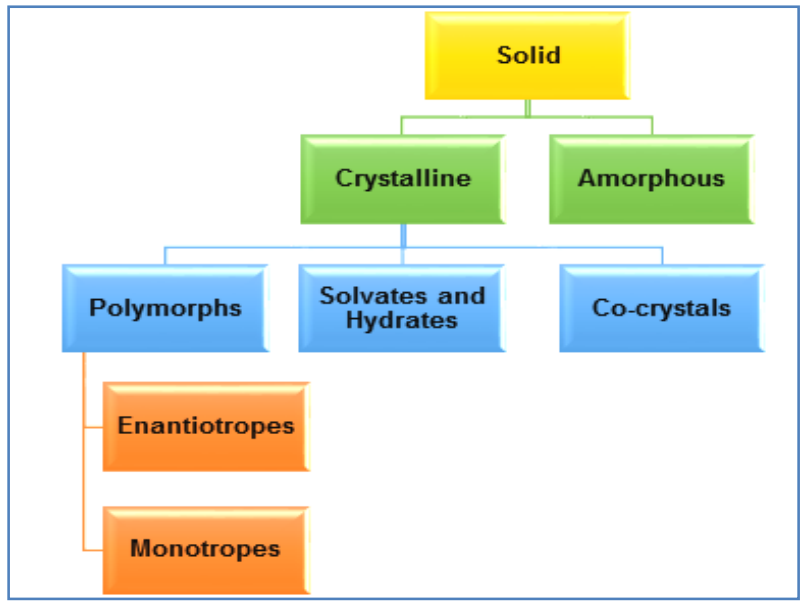

Figure 3: Classification of solid

\section{Properties affected by polymorphism}

This different arrangement creates a difference in the energies of the two polymorphs, hence they differ in their properties such as packing properties, Thermodynamic properties, spectroscopic properties, kinetic properties, surface properties and mechanical properties. Some example of these properties are listed below (Table 1).

Table 1: Physical properties that differ among crystal forms

\begin{tabular}{|l|l|}
\hline Property type & Example \\
\hline Thermodynamic & $\begin{array}{l}\text { Melting point, solubility, heat } \\
\text { capacity, entropy }\end{array}$ \\
\hline Spectroscopic & $\begin{array}{l}\text { Electronic, vibrational and Nuclear } \\
\text { spin transitions }\end{array}$ \\
\hline Packing & $\begin{array}{l}\text { Refractive index, density, Molar } \\
\text { volume, conductivity }\end{array}$ \\
\hline Kinetic & $\begin{array}{l}\text { Dissolution rate, rate of reaction } \\
\text { and stability }\end{array}$ \\
\hline Surface & $\begin{array}{l}\text { Surface free energy, crystal habit, } \\
\text { interfacial tensions }\end{array}$ \\
\hline Mechanical & $\begin{array}{l}\text { Hardness, compactibility, flow and } \\
\text { blending }\end{array}$ \\
\hline
\end{tabular}

Thus, polymorphism can affect the quality, safety, and efficacy of the drug product due to the difference in these physical properties of a different polymorph of the same chemical compound. Hence the detection, identification, quantitation and characterisation of polymorph is very important in an early stage of the drug development which can help to avoid manufacturing defects and improve the quality of drug hence this makes the study of polymorphism very important nowadays as many of drug are formulated in crystalline form.

\section{Regulatory guidelines related to polymorph}

Various regulatory agencies describe the guidelines for the polymorph identification, characterisation and its control in drug substances and drug Product [6-8].

\section{Food drug administration (FDA)}

The United States Food drug administration has published a guidance for industry entitled ANDAs: Pharmaceutical Solid Polymorphism: Chemistry, Manufacturing, and Controls Information in July 2007. This FDA guidance describes the definition of polymorph and polymorphism, the importance of polymorphism, characterisation of polymorphs, the influence of polymorphism on drug substances and drug product, setting a limit of polymorph and decision tree. FDA has also published a guidance for industry for Co-crystals entitled Regulatory Classification of Pharmaceutical Co-Crystals Feb 2018. This guideline provides the guidance for applicants planning to submit new drug applications (NDAs) and abbreviated new drug applications (ANDAs) with information on the appropriate regulatory classification of pharmaceutical co-crystal solid-state forms.

\section{European medicines agency (EMA)}

A similar guideline is also published by the European medicines agency (EMA) titled, Reflection paper on the use of co-crystals of active substances in medicinal products in May 2015. EMA guideline titled Guideline on the chemistry of active substances published in Nov 2016, explains the polymorphism and analytical methods used for its identification.

\section{International Council for Harmonisation (ICH)}

ICH describe the definition of polymorph and polymorphism along it characterisation by various analytical technique and decision tree in its Q6A guideline titled Specifications: Test Procedures and Acceptance Criteria for New Drug Substances and 
New Drug Products: Chemical Substances dated Oct 1999.

\section{Screening of polymorph}

If the transformation of polymorph occurs, then there may be a change in its properties such as stability, dissolution, compatibility during clinical development or after introduction to the market, this might pose a serious risk to patient safety [9].

To minimize this risk, a polymorph screening should always be performed which is required according to the ICH Q6A guidelines which dictate the requirement of a polymorph screening for all pharmaceutical products. Screening has to ensure that the polymorphic form you use in development is sufficiently stable. The $\mathrm{ICH}$ decision trees 1 to 3 shown below provide ANDA applicants with a suggested process for evaluating the importance of and approaches to setting specifications for polymorphic forms in solid oral drug products and oral suspensions.

Decision Tree 1 provides recommendations on when specifications for polymorphic form for the drug substance or the drug product may be appropriate. Polymorphs are unlikely to have a significant effect on Bioavailability (BA) and Bioequivalence (BE) when all forms have the same apparent solubility or all forms are highly soluble.

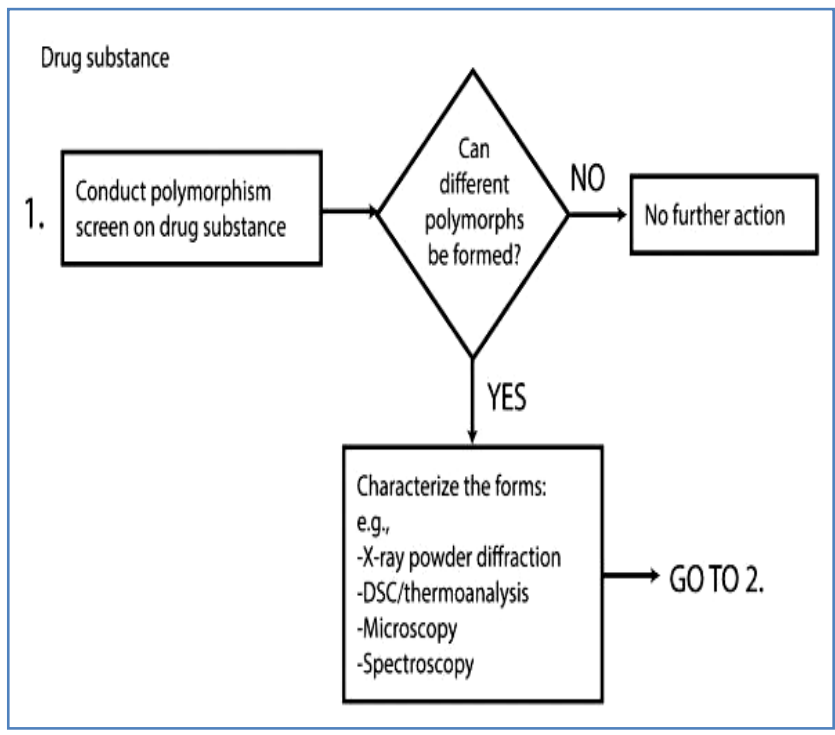

Figure 4: Decision Tree 1

Decision Tree 2 provides an approach for setting specifications for polymorphs in the drug substance when at least one form is known to have low solubility based on the Biopharmaceutics Classification System (BCS).

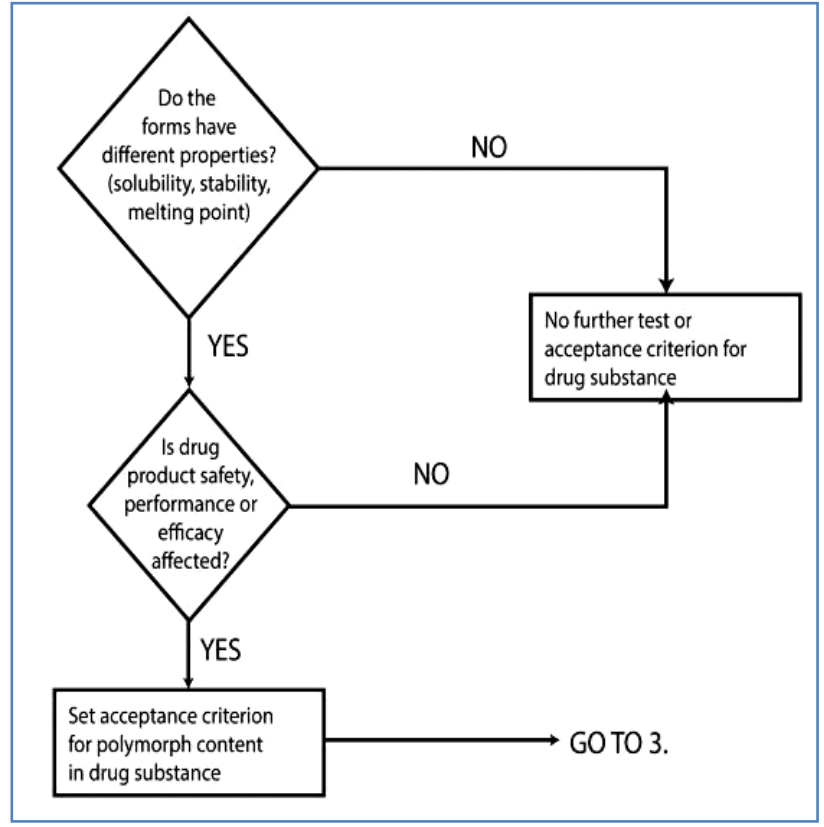

Figure 5: Decision Tree 2

Decision Tree 3 provides an approach when considering whether to set specifications for polymorphs in the drug product. Generally, specifications for polymorphs in drug products are not necessary if the most thermodynamically stable polymorphic form is used.

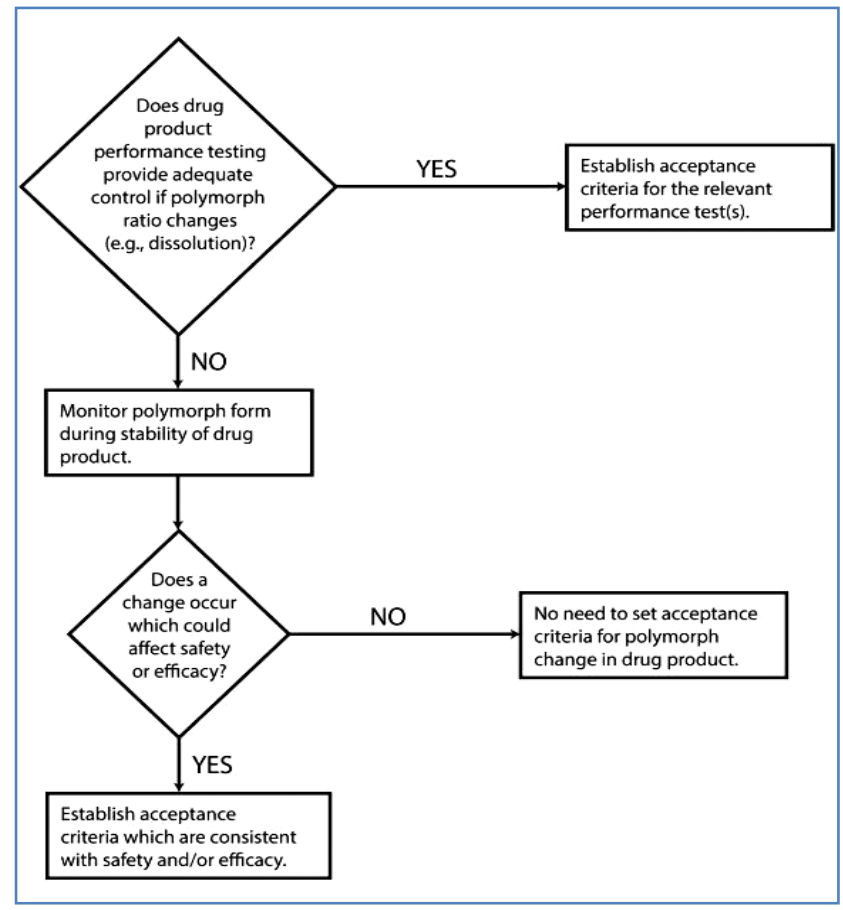

Figure 6: Decision Tree 3

\section{Polymorph affecting the pharmaceuticals}

Polymorphism in drug substances and excipient greatly affect the properties of the drug product 
with respect to its solubility, bioavailability, toxicity and stability [10].

\section{Solubility and Bioavailability}

Formulations, where the drug substances get, dissolves in the body, much attention is to be paid with regards to polymorph as different drug polymorph may have different solubility and bioavailability, hence affect the release of the drug. Control of the unrequired form by the manufacturer is required during the processing of drug substances and excipients in the manufacturing of a drug product.

\section{Stability}

In the manufacturing of drug product, the polymorph which is most stable is used as it has the lowest potential to convert into another form. One polymorph may convert to another during manufacturing and storage, particularly when a metastable form is used. Generally, crystalline forms are more stable when compared to amorphous form. Polymorph may get converted to another form during manufacturing and storage if the metastable form is used.

\section{Toxicity}

Solvates of potentially harmful solvents in the crystal lattice may cause drug substances or excipients may be toxic or harmful to patients should also be suitably controlled and monitored by the manufacturer.

\section{Characterisation of polymorph}

Polymorphs of a drug substance are usually characterized based on the differences in their physical properties some of which are packing properties, thermodynamic properties, spectroscopic properties, kinetic properties, surface properties and mechanical properties. Analytical techniques associated with these properties used for the identification, quantification and characterisation of the polymorph are as follows [11-13].

\section{$X$-ray powder diffraction (XRPD)}

Polymorph have different crystal structure when analysed by X-ray diffractometer give characteristic $X$-ray diffraction pattern having peaks at a certain position and of varying intensities.
The data output of the instrument is a plot of

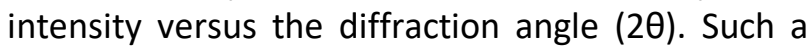
plot can be considered as a fingerprint of the crystal structure of that polymorph. If the material is amorphous then diffraction pattern consists of the broad hump. XRD analysis can be used for qualitative identification or extended to fully quantitative analysis. The analysis is also applicable to a mixture of polymorphs or to multi-component formulations.

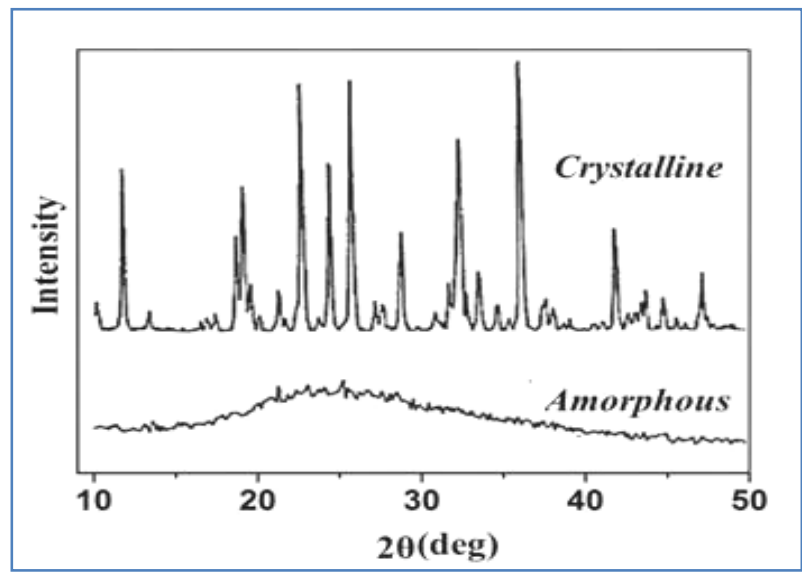

Figure 7: Typical diffractogram of crystalline and amorphous samples

\section{Differential Scanning Calorimetric (DSC)}

All polymorphic transitions involve a change in enthalpy. DSC technique measures temperature and enthalpy (heat) associated with these transitions in the materials. Thus DSC provides quantitative and qualitative information about physical and chemical changes that involve endothermic or exothermic processes, or changes in the heat capacity.

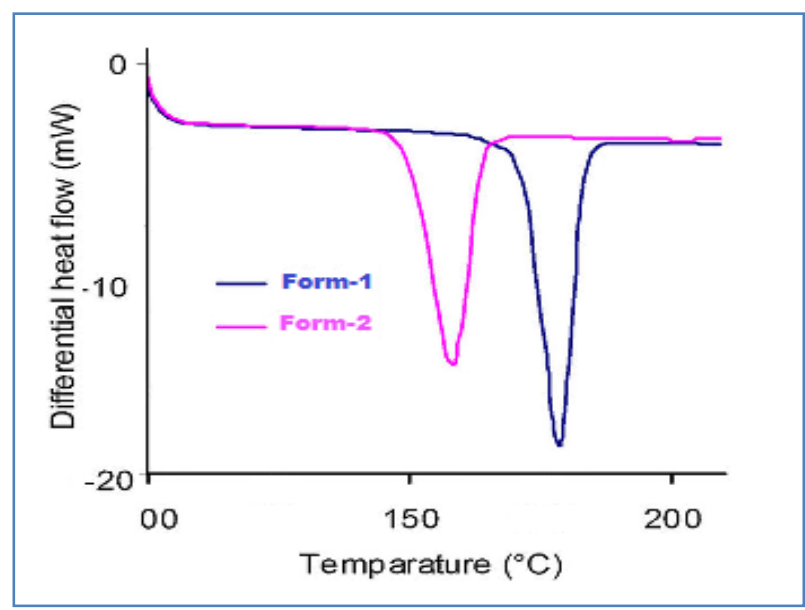

Figure 8: Typical DSC thermogram of two different forms 


\section{Differential Thermal Analysis (DTA)}

It is a technique in which the difference in temperature between the sample and reference material is monitored against time or temperature while the temperature of the sample, in a specified atmosphere, is programmed. It is useful for polymorph undergoing the fusion, boiling, sublimation, vaporization; crystalline structure inversion, solid-solid transition, and water loss.

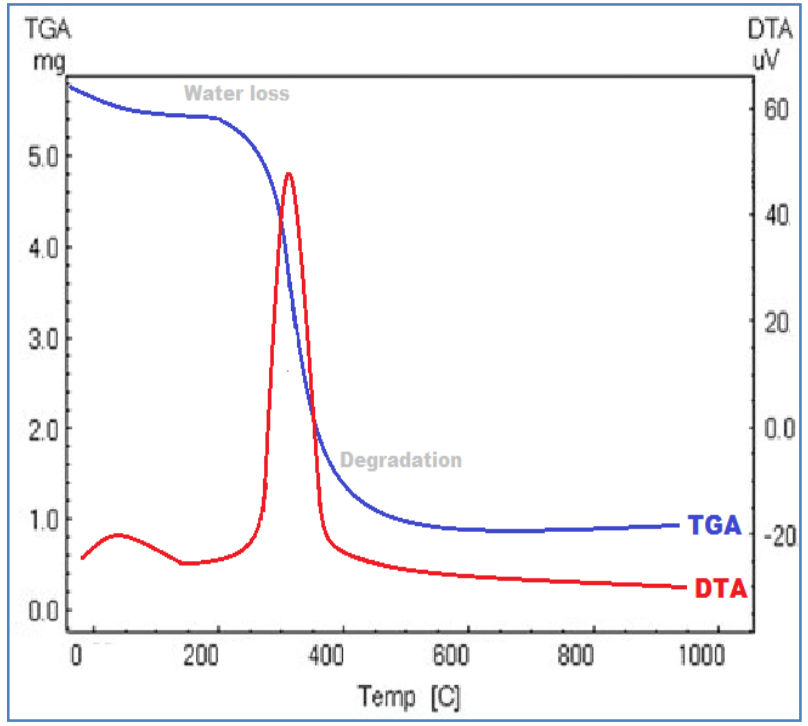

Figure 9: Typical DTA and TGA thermogram

\section{Thermogravimetric analysis (TGA)}

TGA is a thermal analysis technique. Here, the change of the mass of a sample is observed with the change of the temperature. This can also be measured as a function of time at a constant temperature. This technique is used in supportive of the other techniques for hydrate, solvate and co-crystal polymorph to establish the thermic event due to desolvation or dehydration (Fig.-3).

\section{Fourier Transforms Infrared Spectroscopy (FT-IR)}

Infrared spectroscopy measures the light absorbed by different types of vibrations in molecules. As molecular crystals are characterized by vibrations as well as translational oscillations and rotational oscillations, which are hindered by the crystalline structure. Different polymorph crystal structures will give different infrared absorption pattern. Thus polymorph is characterised by FTIR (Fig.-4). Various sample technique such as ATR and DRS can be used for sample preparation. Also, FTIR instrument can also be hyphenated with other thermal technique to study the detail of polymorph.

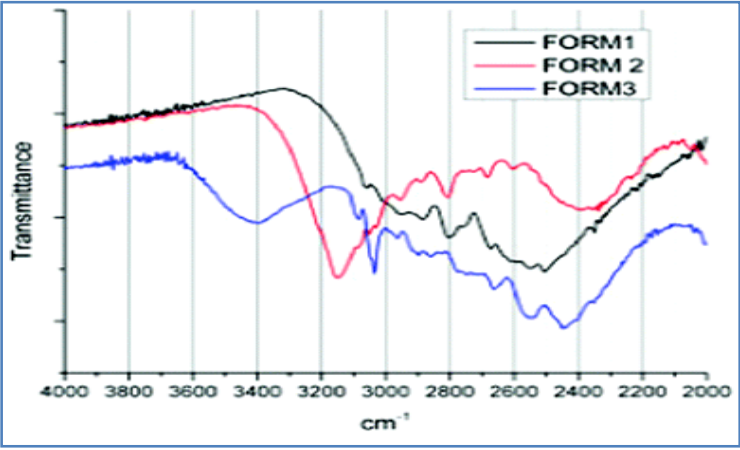

Figure 10: Typical FTIR spectra of polymorphs

\section{Raman Spectroscopy}

Polymorph is identified by detecting scattering light at low wavenumbers derived from lattice vibration by laser irradiation of the sample. Raman spectra of crystals with small differences in bond lengths or crystal spacing will provide small differences in the Raman peak positions. Both crystalline and amorphous structures can be evaluated by this technique. This technique requires no or very less time for sample preparations.

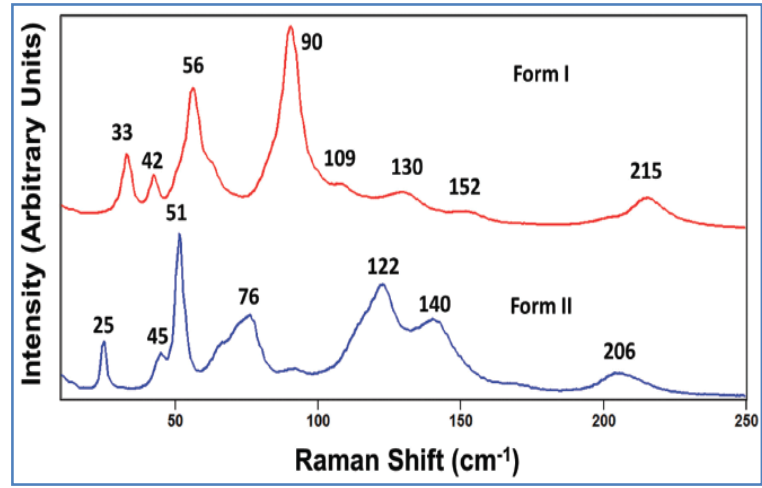

Figure 11: Typical Raman spectra of polymorphs

\section{Solid State NMR Spectroscopy}

In NMR spectroscopy, the nuclei of atoms are irradiated with strong radio waves, and the corresponding resonance frequency of the nuclei is observed. The chemical shift is very sensitive to slight changes in the local electronic environment and so different crystalline forms, whether they are polymorphs, hydrates or solvates, will have different NMR spectra.

Long data acquisition time is the major drawback of this solid-state NMR, so it is not considered for routine analysis of samples. However, it is usually a sensitive technique and sample preparation is minimal. This technique offers little interference from many excipients. 


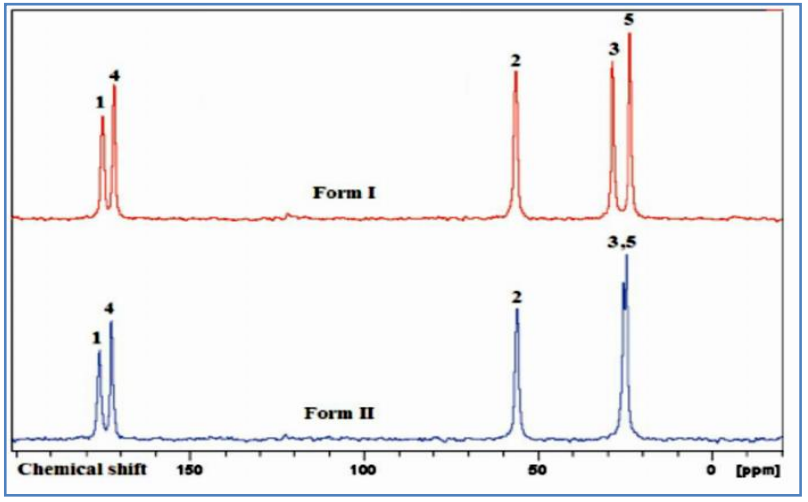

Figure 12: Typical NMR spectra of polymorphs

\section{Optical microscopy}

It consists of a polarizing light microscope capable of taking a photo or recording the event. It is generally used to study solid state properties, crystallinity, refractive index, and particle size \& shape of different polymorph.

\section{Scanning Electron Microscopy}

A scanning electron microscope (SEM) produces images of a sample by scanning the surface with a focused beam of electrons. The electrons interact with atoms in the sample, producing various signals that contain information about the surface topography and composition including morphology, polymorph identification, mapping of crystal habit to crystal structure and crystal defect characterization. Scanning Electron Microscopy has two distinct advantages over common optical microscopy - namely, much higher magnification and greater depth of field.

\section{Hot Stage Microscopy}

It is a combination of microscopy and thermal analysis, also known as thermal microscopy (TM) This technique enables the study of physical characterization of polymorphs and solvates as a function of temperature and time. This technique is simple and relatively inexpensive since the instrument setup basically comprises of a sample holder with heating furnace and inner gaseous atmosphere having a suitable polarized-light microscope along with a system that allows the capturing and measurements of observations and temperatures.

\section{Factors affecting polymorphism}

There are various factor which appears to affect the drug substance and drug product polymorphism. Some of these factor are describe below [14].

\section{Temperature and humidity}

Drug substances and drug product on storage at a higher temperature accelerate the physicochemical reactions according to activation theory of Arrhenius. Exposure to environmental conditions such as humidity and temperature can also induce polymorph conversion. Humidity present at surface acts as a catalyst and assist the degradation of the product. The extent of conversion generally depends on the relative stability of the polymorphs, kinetic barriers to phase conversion, and applied stress. Hence control of temperature during the manufacturing and storage of a drug substance and drug product is very necessary.

\section{Effect of grinding}

The process of grinding reduces the particle size to the nanoscale. Polymorphs that are metastable at the micrometre or larger size crystals can be converted by grinding to nanoscale which may be thermodynamically stable form. Grinding of material increasing the specific surface area which directly affects the rate of dissolution and bioavailability. This behaviour may be explained simply by a difference in the energy barrier for the transition from one form to another. Precaution is to be taken that with increasing grinding time compound become unstable because grinding weakened bonding crystals and water molecules participating in the hydrolysis process of the drug.

\section{Photostability}

Different polymorph may have different photochemical reactivity upon exposure to photo light. The arrangement of atoms in the solid state structure of the two forms of has a profound effect on resultant photo light stability. Thus upon exposure, to photo light metastable form gets converted to the stable form, hence they show different photostability. Therefore these lightsensitive drugs are protected from the photolytic degradation by packing them suitably in a lightresistant container.

\section{Effect of solvent}

In solution, various clusters are formed due to the intermolecular interaction of solute and solvents. This interaction brings a short range of order in the 
solution which increases with time to form a larger crystal lattice. Thus, various types of solvent can bring various types of order which give rise to polymorphic crystal. Similarly, various crystallization process such as solvent evaporation, slow cooling solvent diffusion, vapour diffusion, vacuum sublimation, etc. forms different polymorphs. Nature of a solvent such as dipolar, aprotic, protic, Lewis-acidic, lewis-basic, aromatic or non-polar solvent can form different polymorphs in the solution form. Incorporation of solvents or water in the crystal lattice of the drug compound in stoichiometric proportions lead to polymorphs such as solvates and hydrates.

\section{Effect of compression}

In the manufacturing of tablet, the compression pressure applied for the formation of the tablet is in the range of 40 to $200 \mathrm{MPa}$ for short time these compression does not induce the phase transformation in major of pharmaceutical. But at high pressure, it brings out phase transformation in the crystal which lead to polymorph formation. Although these small but significant number of pharmaceutical are known to undergo transformation upon compression during the manufacturing process of the drug product.

\section{Conclusion}

Study of polymorphism and polymorph characterisation is very important for the manufacturing of quality and stable drug substances and drug product. As different polymorph have different properties such as packing properties, thermodynamic properties, spectroscopic properties, kinetic properties, surface properties and mechanical properties hence correct property lead to required bioavailability of the drug. Different advance analytical techniques such as thermal, spectroscopic and image analysis used for the detection, quantification and characterisation of the polymorphs help in its control in the drug substances and drug products.

\section{References}

1. Schütt, H. W. (1997). Eilhard Mitscherlich, prince of Prussian chemistry. Chemical Heritage Foundation.
2. Lee, E. H. (2014). A practical guide to pharmaceutical polymorph screening \& selection. asian journal of pharmaceutical sciences, 9(4), 163175.

3. Bond, A. D., Boese, R., \& Desiraju, G. R. (2007). On the polymorphism of aspirin: crystalline aspirin as intergrowths of two "polymorphic" domains. Angewandte Chemie International Edition, 46(4), 618-622.

4. Brittain, H. G. (Ed.). (2016). Polymorphism in pharmaceutical solids. CRC Press.

5. Hilfiker, R. (Ed.). (2006). Polymorphism in the pharmaceutical industry (Vol. 2, pp. 1-20). Weinheim: Wiley-Vch.

6. Food and Drug Administration. (2007). Guidance for industry. ANDAs: pharmaceutical solid polymorphism. Chemistry, manufacturing and controls information. Center for Drug Evaluation and Research.

7. Kumar, A., Kumar, S., \& Nanda, A. (2018). A review about regulatory status and recent patents of pharmaceutical co-crystals. Advanced pharmaceutical bulletin, 8(3), 355.

8. Elder, D. (2017). ICH Q6A Specifications: test procedures and acceptance criteria for new drug substances and new drug products: chemical substances. ICH Quality Guidelines: An Implementation Guide, 433-466.

9. Guideline, I. C. H. (1999). Q6A. Specifications: test procedures and acceptance criteria for new drug substances and new drugs products: chemical substances.

10. Lee, A. Y., Erdemir, D., \& Myerson, A. S. (2011). Crystal polymorphism in chemical process development. Annual review of chemical and biomolecular engineering, 2, 259-280.

11. Giron, D. (2002). Applications of thermal analysis and coupled techniques in pharmaceutical industry. Journal of thermal analysis and Calorimetry, 68(2), 335-357.

12. Bugay, D. E. (2001). Characterization of the solidstate: spectroscopic techniques. Advanced Drug Delivery Reviews, 48(1), 43-65.

13. Yu, L., Reutzel, S. M., \& Stephenson, G. A. (1998). Physical characterization of polymorphic drugs: an integrated characterization strategy. Pharmaceutical Science \& Technology Today, 1(3), 118-127.

14. Kitamura, M. (2002). Controlling factor of polymorphism in crystallization process. Journal of Crystal Growth, 237, 2205-2214. 